\title{
A new method for studying the thermospheric density variability derived from CHAMP/STAR accelerometer data for magnetically active conditions
}

\author{
M. Menvielle ${ }^{1,2}$, C. Lathuillère ${ }^{3}$, S. Bruinsma ${ }^{4}$, and R. Viereck ${ }^{5}$ \\ ${ }^{1}$ CNRS/IPSL, Université Versailles St-Quentin, Centre d'études des Environnements Terrestre et Planétaires, UMR 8639, \\ 94107 Saint Maur Cedex, France \\ ${ }^{2}$ Univ. Paris Sud, 91405 Orsay Cedex, France \\ ${ }^{3}$ Laboratoire de Planétologie de Grenoble, UJF, CNRS, BP 53, 38041 Grenoble Cedex 9, France \\ ${ }^{4}$ CNES, dept. of Terrestrial and Planetary Geodesy, 31401 Toulouse Cedex 4, France \\ ${ }^{5}$ NOAA Space Environment Centre, 325 Broadway, Boulder, CO 80305-3328, USA
}

Received: 8 November 2006 - Revised: 17 July 2007 - Accepted: 10 August 2007 - Published: 2 October 2007

\begin{abstract}
Thermospheric densities deduced from STAR accelerometer measurements onboard the CHAMP satellite are used to characterize the thermosphere and its response to space weather events. The STAR thermospheric density estimates are analysed using a Singular Value Decomposition (SVD) approach allowing one to decouple large scale spatial and temporal variations from fast and local transients. Because SVD achieves such decomposition by using the reproducibility of orbital variations, it provides more meaningful results than any method based upon data smoothing or filtering.

SVD analysis enables us to propose a new thermosphere
\end{abstract} proxy, based on the projection coefficient of the CHAMP densities on the first singular vector. The large scale spatial variations in the density, mostly related to altitude/latitude variations are captured by the first singular vector; time variations are captured by the associated projection coefficient.

The study presented here is focused on time dependent global scale variations in the thermospheric density between $50 \mathrm{~N}$ and $50 \mathrm{~S}$ geographic latitudes. We show that the time variations in the projection coefficient do in fact represent those in the global density that are associated with magnetic activity as well as with solar EUV radiations. We also show that the NRLMSISE-00 empirical model better accounts for the density forcing by Solar radiations when tuned using $\mathrm{Mg}$ II indices. Using the so modified model with an additional geomagnetic parameterization corresponding to quiet geomagnetic situation enables one to define time reference values which are then used to evaluate the impact of geomagnetic activity. The ratio of CHAMP density projection coefficient to the quiet model projection coefficient is a global

Correspondence to: M. Menvielle

(michel.menvielle@cetp.ipsl.fr) quantity, independent of altitude and latitude, which quantifies the thermospheric density response to auroral energy deposition. It will serve as a proxy of the response of thermospheric density to geomagnetic activity forcing.

Keywords. Magnetospheric physics (Solar windmagnetosphere interactions) - Atmospheric composition and structure (Pressure, density, and temperature; Instruments and techniques)

\section{Introduction}

The CHAMP satellite was launched in July 2000 in a nearcircular orbit at about $450 \mathrm{~km}$ altitude with an inclination of $87.3^{\circ}$ (Reigber et al, 2002). The high precision and stability of the STAR accelerometer installed within one mm of its centre of mass, and the high precision of GPS tracking, allowed significant progress in the precision of thermospheric density retrieval to be made. The resulting accuracy of thermospheric density values derived from STAR accelerometer measurements makes it possible to study the statistical properties of the thermospheric density global distribution (Bruinsma et al., 2004; Liu et al., 2005), or its response to the geomagnetic activity forcing, in particular during severe geomagnetic storms (Sutton et al., 2005; Forbes et al., 2006; Bruinsma et al., 2006).

In a previous paper (Lathuillère and Menvielle, 2004), we studied the thermospheric temperature disturbance due to auroral energy deposition. Using temperatures deduced from WINDII interferometer measurements during magnetically active days, we derived a quantitative description of the thermospheric temperature forcing by geomagnetic activity and

Published by Copernicus Publications on behalf of the European Geosciences Union. 
found evidence of a large underestimation of the model temperatures, even during periods of moderate magnetic activity.

In the present paper, we consider the possibility of using the CHAMP densities for a statistical analysis of the thermospheric density forcing by geomagnetic activity. We are thus interested in variations in the thermospheric densities and not in their absolute values. Accordingly, we do not have to deal with systematic constant errors in the density derivation, such as those resulting from, e.g., uncertainties in the drag coefficients used to derive density from satellite acceleration. On the contrary, we have to pay due attention to errors in the density estimates due to the thermospheric wind action on the satellite and to errors that result from the variation of the satellite altitude

Thermospheric winds are sensitive to geomagnetic activity particularly in auroral regions during periods of magnetic storms, but they do not significantly vary during magnetically quiet periods. Bruinsma et al. (2004) showed that the density determination error due to winds is always less than $5 \%$ when geomagnetic activity is low or moderate $\left(K_{p} \leq 3\right.$ or $\mathrm{am} \leq 40 \mathrm{nT}$ ). Forbes et al. (2006) found that these errors may be $30 \%$ or even more at high latitudes during magnetically disturbed periods, but at latitudes below $50^{\circ}$, density errors are evaluated not to be larger than $10 \%$ and not to vary so much with magnetic activity. In what follows, we therefore limit our analysis to low latitudes between $50 \mathrm{~N}$ and $50 \mathrm{~S}$.

The altitude of the satellite varies by about $40 \mathrm{~km}$ between the perigee and apogee, and its mean altitude varies by tens of kilometres over one year (for example, it varies between 390 and $460 \mathrm{~km}$ during the year 2002). Using CHAMP thermospheric densities for characterizing the thermospheric perturbations associated to magnetic activity therefore requires us to compensate for the density variations related to changes in the satellite altitude.

The usual solution for achieving this objective is to normalize the CHAMP densities by means of projection to a common altitude. Such procedure actually implies projection of the density values over an altitude range that can reach one thermosphere scale height. In practice, the scale height used for such normalization is estimated by means of empirical thermosphere models. Such models tend to underestimate the disturbances in the exospheric temperature in presence of magnetic activity (Burns et al., 1995; Killeen et al., 1995), by as much as $70 \%$ (Lathuillère and Menvielle, 2004) which in turn leads to errors in scale height that can reach $15 \%$, and errors in the normalized densities (over one scale height) of the same order of magnitude. These errors depend on the geomagnetic activity through the model imperfectness, which introduce unknown biases to the normalized densities.

Another solution is to get rid of the density variations related to changes in the satellite altitude by defining a reference that makes it possible to compare observations made at different satellite positions, i.e. at different altitudes, latitudes and local times LT. The orbit LT drift is of few minutes per $24 \mathrm{~h}$; during the same period, the altitude of the satel- lite varies by less than few hundreds of meters at a given latitude. During periods of magnetic quietness, density variations along $50 \mathrm{~N}-50 \mathrm{~S}$ orbit segments at almost the same LT are expected to be very similar. A method such as the Singular Value Decomposition (SVD) that uses the reproducibility of density variations along successive orbits is well suited to extract the altitude/latitude/LT reference profile from $24 \mathrm{~h}$ data.

We therefore consider using SVD to get rid of scaleheight modelling in thermospheric density characterization. In Sect. 2, the SVD method is briefly described, and the 15orbit "running SVD method" we use is presented, and illustrated by the analysis of the May 2003 CHAMP density data series. In particular, we show that this method enables one to derive a global coefficient that gives an overview of the thermosphere behaviour at middle and low latitudes. The same SVD procedure is used to analyze thermospheric density computed at the satellite position with the NRLMSISE00 empirical model (Sect. 3). Computations are made for actual magnetic conditions on the one hand, and for quiet ones on the other hand. The comparison between computed NRLMSISE-00 and observed CHAMP densities makes it clear that using the Mg II index as a proxy for solar EUV instead of the classical F10.7 index leads to a better description of the thermospheric density. It is shown that SVD analysis of the densities computed for quiet magnetic conditions can be used to derive a reference level for studying thermospheric density perturbations in response to space weather events.

\section{SVD analysis of CHAMP densities during May 2003}

We present here the analysis of a one-month period: 1 to 31 May 2003, which ends with a large storm studied in Hanuise et al. (2006). We use the total density estimates derived from CHAMP STAR accelerometer measurements sampled at $10 \mathrm{~s}$ interval, and interpolate them to the nearest degree latitude value.

The reduction of the CHAMP/STAR accelerometer data in terms of total density is described in detail in Bruinsma et al. (2004), and we refer the reader to this publication for an extensive description of the CHAMP density determination.

Let us just recall here that the errors in the thermospheric density variations due to neglecting winds remain smaller than $5 \%$ during magnetic magnetically quiet periods in the $50 \mathrm{~N}$ to $50 \mathrm{~S}$ latitude interval we consider in this work. In this latitude range, this error may reach $10 \%$ during large storms, which is small compared to the $200 \%$ or larger observed density enhancements during such large magnetic storms (Sutton et al., 2005; Forbes et al., 2006; Bruinsma et al., 2006).

The left panel of Fig. 1 shows the latitude - local time coverage of the data on 2 May and 29 May; the direction of the satellite motion along its orbit is shown by the arrows. The local time does not significantly vary during any day for each of the North-South, and the South-North orbit segments 
between $50 \mathrm{~N}$ and $50 \mathrm{~S}$. Local time variation over the whole month is less than $3 \mathrm{~h}$, from 5.6 to 03:00 LT for the NS segment and from 17.6 to 15:00 LT for the SN segment. The altitude of the $50 \mathrm{~N}$ to $50 \mathrm{~S}$ segments of the two first orbits of 2 and 29 May are shown on the right panel of Fig. 1. Their altitude variation is about $20 \mathrm{~km}$. This plot also shows that at given latitude the altitude variation over the month is no more than $3 \mathrm{~km}$ for the NS night time segment, and much less for the $\mathrm{SN}$ day time segment.

This illustrates the fact that, during any UT 24-h interval, the SN (respectively NS) orbit segments have almost the same LT and very similar latitude versus altitude profile. Because of the $12 \mathrm{~h}$ difference in LT between them, NS and SN segments will be considered separately.

During magnetically quiet periods, the density versus latitude profile is then expected not to change significantly from one orbit NS (or SN) segment to the other during any 24-h UT interval. Hence the reproducibility of the density variations that are related to latitude and altitude changes along the orbital path should allow decoupling fast and local transients from the signal related to orbital modulation. A method such as the Singular Value Decomposition (SVD) that uses the reproducibility of density variations along successive orbits is well suited to extract the altitude/latitude/LT reference profile from the density variations observed along the 15 consecutive orbits corresponding to a given $24 \mathrm{~h}$ time window.

\subsection{The 15-orbit running SVD analysis method}

The mathematical bases of the Singular Value Decomposition are briefly recalled in the appendix. We refer the reader to classical linear algebra treaties for demonstrations, and further mathematical developments (see, e.g., Horn and Johnson, 1985).

In what follows, the SVD analysis is made on the set of 15 orbit segments (NS or SN ones) corresponding to the considered orbit, the 7 previous ones and the 7 following ones. This is somehow a 15 -orbit running SVD analysis over 15 consecutive orbit sections, i.e. a 24-h long time window. The first normalized singular value, called in the following the $\lambda_{1}$ parameter, is always found to be larger than 0.98 . This suggests that the thermosphere density variations related to orbital modulation are captured by the first singular vector $\boldsymbol{u}_{1}$. It is actually what is observed, as illustrated by Fig. 2 .

Let $\boldsymbol{d}$ the vector whose elements $d\left(\varphi_{j}\right)$ are the density observed along the considered orbit at latitude $\varphi_{j}$ : the dimension of $\boldsymbol{d}$ is 101 , corresponding to the 101 latitude degrees between $50 \mathrm{~N}$ and $50 \mathrm{~S}$. We therefore express $\boldsymbol{d}(\phi)$ as:

$\boldsymbol{d}(\phi)=c_{1} \boldsymbol{u}_{1}+\boldsymbol{r}_{1}=\delta_{1}(\phi)+\boldsymbol{r}_{1}$

where $\boldsymbol{r}_{1}$ is the residual that captures fast and local transients along the considered orbit, $c_{1} \boldsymbol{u}_{1}$ is the projection of the observed density profile $\boldsymbol{d}$ on the singular vector $\boldsymbol{u}_{1} ; c_{1}$ has the dimension of a density. Note that the definition of $c_{1}$ im-
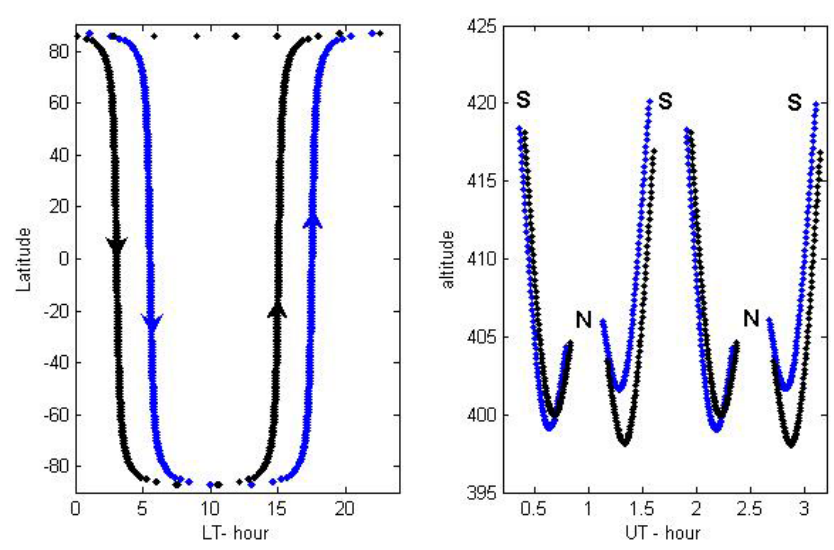

Fig. 1. Left panel: latitude - local time coverage of the data on 2 May (black diamonds) and 29 May (blue diamonds); the direction of the satellite motion along its orbit is shown by the arrows.

Right panel: altitude of the $50 \mathrm{~N}$ to $50 \mathrm{~S}$ segments of the two first orbits of 2 and 29 May; the $\mathrm{N}$ and $\mathrm{S}$ letters refer to the $50 \mathrm{~N}$ and $50 \mathrm{~S}$ ends of the orbit segments, respectively.

plies that its typical value is ten times larger than the typical density value along the orbit segment.

Figure 2 presents the results of the 15-orbit SVD analysis for one orbit during magnetically quiet period (5 May between $\sim 01: 40$ and $\sim 02: 50 \mathrm{UT}, \mathrm{am}=9 \mathrm{nT}$; left panel) and one orbit during magnetic activity ( 22 May between $\sim 11: 50$ and $\sim$ 13:00 UT, am $=46 \mathrm{nT}$; right panel). In both cases, the $\lambda_{1}$ parameter is larger than 0.995 . Figure 2 illustrates how $\boldsymbol{\delta}_{1}$ captures the orbital modulation. It is worth noting here that $\delta_{1}$, and then $c_{1}$ are smaller during quiet periods than during magnetic activity. Figure 2 also illustrates the fact that the residual $\boldsymbol{r}_{1}$ is negligible during magnetically quiet periods, while it significantly increases in presence of magnetic activity. During magnetic storm the residual may reach values on the order of those of the densities observed during magnetically quiet periods (not shown).

\subsection{The SVD analysis of the May 2003 CHAMP density}

Figure 3 displays, as a function of UT time (each orbit segment being associated to the universal time of the equator crossing.), the $\lambda_{1}$ parameter (upper panel), the $c_{1}$ projection coefficient (middle panel), and both the Solar F10.7 and geomagnetic $a_{p}$ indices (lower panel). The results for day time $(\mathrm{SN})$ and night time (NS) orbit segments are displayed.

The singular values varies between 0.99 and 1, meaning, that almost all the energy of the density variations is captured by the projection on the first singular vector, as shown by the following colour plots. For day time data, this $\lambda_{1}$ parameter drops only during the large storm that occurs at the end of the month (day 30), while for night time data it also decreases during periods of lower magnetic activity (days 512). The $c_{1}$ curves corresponding to night time and day time orbit segments have variations that appear to be correlated to 

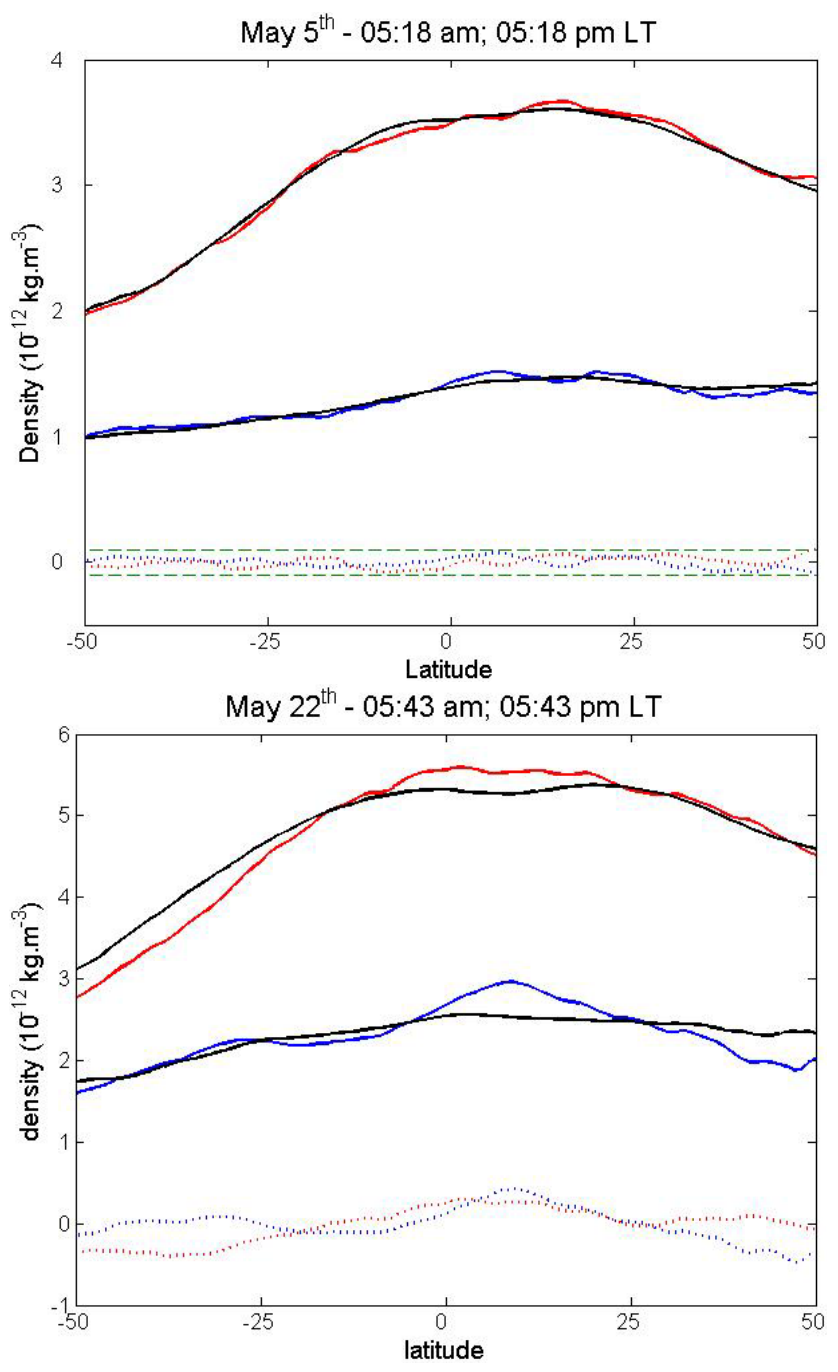

Fig. 2. Example of results of the 15-orbit SVD analysis. The observed density variations along the NS and SN orbit segments $(\boldsymbol{d}(\varphi)$; red line for day time. blue line for night time) are plotted together with their projection along the direction of the first singular vector $\left(\boldsymbol{\delta}_{1}(\varphi)\right.$; black line). The residual $\boldsymbol{r}_{1}$, i.e. the difference $\boldsymbol{d}(\varphi)-\boldsymbol{\delta}_{1}(\varphi)$ is also plotted (dotted lines; red for day time, blue for night time). See Eq. (A1) and text for further explanation.

Upper panel: case of one orbit during magnetically quiet periods (5 May, am=9 nT); Lower panel: case of one orbit during magnetic activity (22 May, am=46 nT).

Note the change in the vertical scale between upper and lower panels: the dashed green lines correspond to densities equal to +0.1 and $-0.110^{-12} \mathrm{~kg} \mathrm{~m}^{-3}$. In both cases, $\boldsymbol{\delta}_{1}(\varphi)$ accounts for long space wavelength features in the observed density variations $\boldsymbol{d}(\varphi)$. The residual is negligible during magnetically quiet periods, while its amplitude may become significant in presence of magnetic activity.

both geomagnetic and Solar activities as monitored by the $a_{p}$ and F10.7 indices, respectively. The $c_{1}$ variations are found
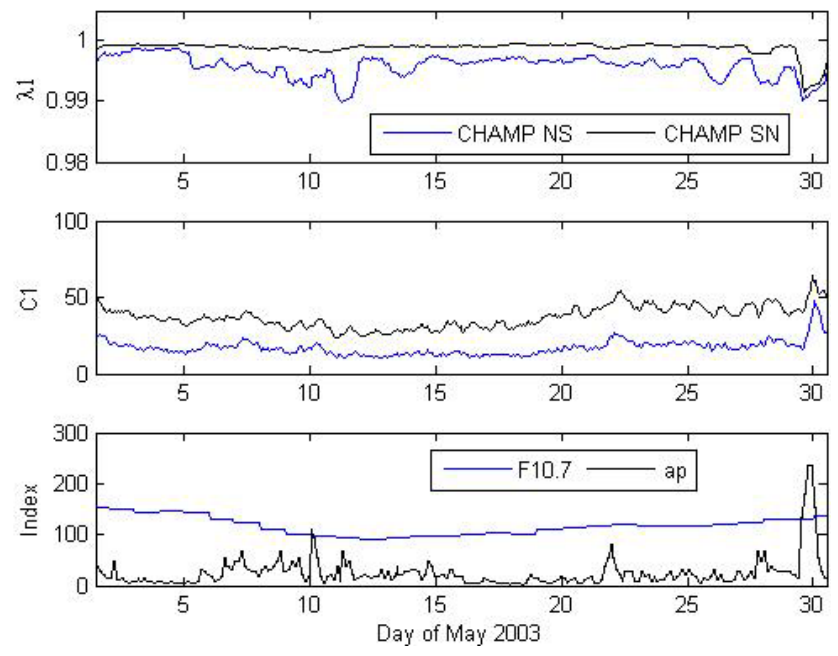

Fig. 3. Variations with UT of the $\lambda_{1}$ parameter (upper panel) and the $c_{1}$ projection coefficient in $10^{12} \mathrm{~kg} \mathrm{~m}^{-3}$ (middle panel) during May 2003, for day time (SN; black line) and night time (NS; blue line) orbit segments. Note that the definition of $\mathrm{c}_{1}$ (see Eq. A1 and text for further details) implies that its value is typically ten times larger than the typical density value along the orbit segment. The variations of the Solar F10.7 (blue line) and geomagnetic $a_{p}$ indices (black line) during May 2003 are displayed in the lower panel.

to be larger during day time, excepted during the large storm of 29 May. Time variations of the order of a day are also clearly visible.

Figures $4 \mathrm{a}$ and $\mathrm{b}$ (upper panels) display the original data corresponding respectively to the NS and SN segments, as a function of latitude and UT time during May 2003. Remember that, in fact, altitude and latitude variations are combined in this colour plot. The density is much larger in the day time (SN) as compared to night time (NS), which is consistent with solar heating of the dayside. One can see also larger densities at middle latitudes in the north, resulting from a combined effect of the satellite altitude variation and of the seasonal variation. A significant UT time dependence of the density over this month period is clearly visible at all latitudes. This time variation includes the effects of solar and magnetic activity. In particular, the storm of 30 May appears as a large increase in densities at all latitudes.

The middle panels of Figs. $4 a$ and $b$ show the projection of the original data on the first singular vector, and residuals are displayed on the bottom panels. One can see that this projection capture the large scale density variation with latitude/altitude. Time variations are very well reproduced even at scale smaller than the day. The small spatial scale variations visible in the residuals are due to wave-like perturbations (see lower panels of Figs. 4a and b). They can be large, as during the storm at the end of the month.

The analysis of the CHAMP densities during May 2003 clearly shows that $c_{1}$ and $\delta_{1}(\varphi)$ provides a description of the global scale thermospheric density variations along orbit 



Fig. 4a. Upper panel: CHAMP/STAR density estimates along the NS orbit segments, as a function of latitude and UT time during May 2003. Remember that, in fact, altitude and latitude variations are combined in this colour plot.

Middle panel: projection of the CHAMP/STAR density data on the first singular vector (see text for further explanation).

Lower panels: residuals, i.e. differences between the CHAMP/STAR densities and their projection on the first singular vector.

During May 2003 and along the NS orbit segments, LT at the satellite position varies from 05:40 a.m. to 02:55 a.m. (see Fig. 1). For the three panels, the density variations are displayed using the colour scale displayed on the right; they are expressed in terms of $10^{-12} \mathrm{~kg} \mathrm{~m}^{-3}$ units.

segments for latitudes between $50 \mathrm{~N}$ and $50 \mathrm{~S}$, in response to the solar radiation and geomagnetic activity forcing.

\section{The SVD analysis of NRLMSISE-00 densities}

Monitoring the response of the atmosphere to the sole geomagnetic activity forcing requires to get rid of all other density variations, and in particular the one associated to UT change of solar activity forcing. In order to assess how to achieve this objective, empirical thermosphere models are used because, despite their known limitations, they provide efficient tools for investigating the response of the thermospheric density to the different driving quantities.

Densities have thus been calculated using the empirical NRLMSISE-00 model (Picone et al., 2002), at the same location (altitude, latitude, longitude) and the same universal time as the CHAMP data, only varying the geomagnetic activity parameters: the first prediction used the model driven by the actual geomagnetic parameters ("actual model"), whereas the second prediction was obtained with a constant daily $A_{p}$ of 4 ("quiet model"). This procedure has already been used in Lathuillère and Menvielle (2004) in order to derive the
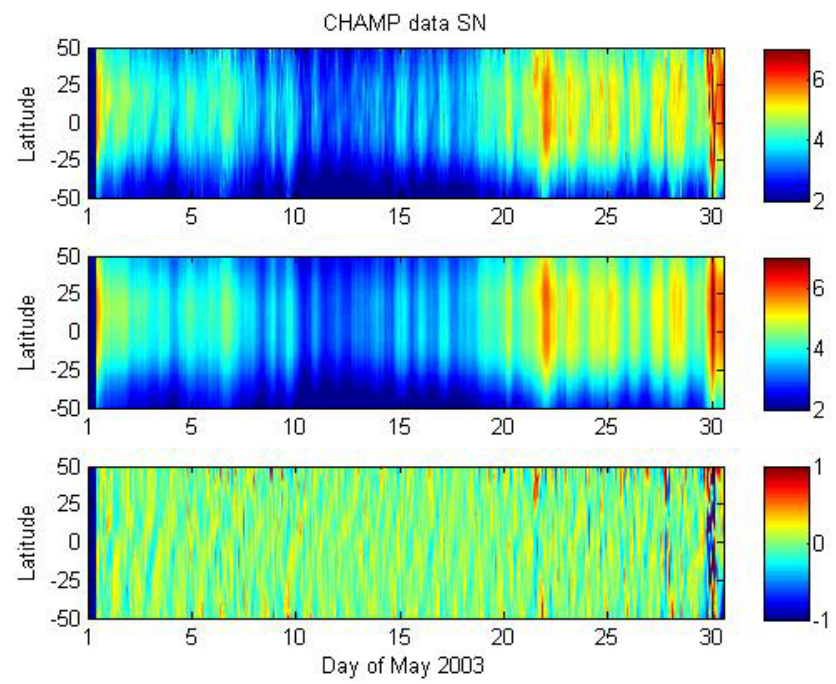

Fig. 4b. Same as Fig. 4a for the SN orbit segments. In this case LT at the satellite position varies from 05:40 p.m. to 02:55 p.m. (see Fig. 1).

thermospheric temperature perturbations due to magnetic activity.

\subsection{The example of May 2003}

The running SVD analysis has been performed on the quiet and actual model densities obtained during the whole month of May 2003, using the same procedure than for CHAMP data.

The variations of the $\lambda_{1}$ parameters have not been plotted as they are equal to 1 , thus indicating that the projection on the first singular vector capture all the time and spatial variations of the thermospheric density as described by the actual and quiet models: the latitude versus altitude dependence is contained within the first singular vector, and the variation with time of the average density value along the orbit segment is described by the $c_{1}$ projection coefficient. This is also true for the actual model during period of high activity. Indeed this is related to the non existence of small scale spatial and time variations, such as, e.g., gravity waves that are smoothed out in the course of the statistical derivation of empirical models from data sets.

Figure 5 presents the $c_{1}$ projection coefficients of the actual (black curves) and quiet (blue curves) models for the night time NS (upper panel) and day time SN (bottom panel) segments.

For the quiet model, the projection coefficients have long term time variations that are very well correlated to those of F10.7 (bottom panel of Fig. 3). These variations also include the effect of the two hour local time shift of the CHAMP orbit between the beginning and the end of May 2003. A small amplitude variation with apparent period about $24 \mathrm{~h}$ is also 



Fig. 5. Variations with UT of the $c_{1}$ projection coefficients for the densities estimated from the CHAMP/STAR observations (thin red lines), and for those computed using the actual (black lines) and quiet (blue lines) NRLMSISE-00 models (see text for the definition of the NRLMSISE-00 actual and quiet models). Upper panel: night time NS orbit segments; lower panel: day time SN orbit segments.

present: it corresponds to the longitude (or equivalently UT) thermospheric density dependence included in the model.

For the actual model, the $c_{1}$ coefficient has short term time variations, superimposed to long term variations similar to those observed for the quiet model one; the short term variations are well correlated to those of the geomagnetic activity as described by the $a_{p}$ index (bottom panel of Fig. 3).

Compare now the results for the quiet and actual model densities with those obtained for the CHAMP densities (thin red lines). The short term time variations correlated with those of the $a_{p}$ index are present in both actual model and CHAMP $c_{1}$ curves. These $a_{p}$-correlated variations are much smaller in the $c_{1}$ actual model curve than in the CHAMP one. The large differences observed in the amplitude of these variations support the fact that empirical thermosphere models do significantly underestimate the variations in thermospheric density driven by geomagnetic activity.

\subsection{Model tuning with the Mg II index}

Another point worth noting is that the quiet model $c_{1}$ values are found to be significantly larger than those of CHAMP at many times and in particular during the period 1 May to 5 May. This is not consistent with the definition of the quiet model. The thermospheric density should in fact be lower in absence of heating by geomagnetic activity. We interpret this in terms of a bias in the representation of the solar activity in the model for this period. This is supported by the comparison presented in Fig. 6 between the F10.7 Solar index and the composite Mg II index described in Viereck et

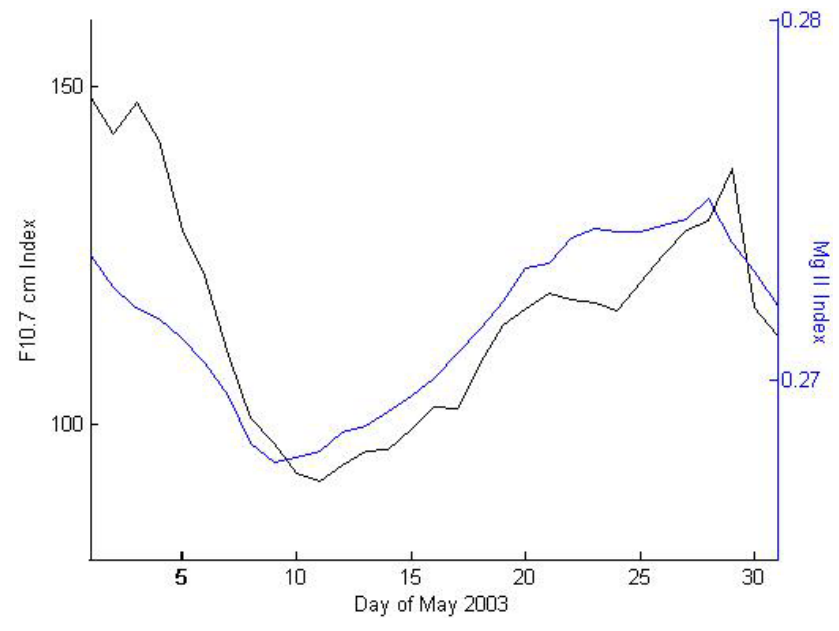

Fig. 6. Variations with UT of the F10.7 Solar index (black line) and the composite Mg II index described in Viereck et al. (2004) (blue line).

al. (2004). The F10.7 index used in the model is plotted as a black line, and the Mg II index by a blue line. At the beginning of the month F10.7 is quite high, and larger than at the end of the month, while the $\mathrm{Mg}$ II index has an opposite behaviour. Using Mg II instead of F10.7 index would therefore lead to smaller quiet model values for the densities and accordingly for the $c_{1}$ projection coefficient at the beginning of the month.

This strongly suggest that using Mg II as a proxy for solar EUV instead of F10.7 in the NRLMSISE-00 model would have been more appropriate to account for the variations in the thermospheric density resulting from the solar EUV forcing during May 2003. This is in accordance with the conclusions of other studies that have already shown that the $\mathrm{Mg}$ II index is a better driver than the F10.7 index for thermospheric models (Thuillier and Bruinsma, 2001; Rhoden et al., 2000; Viereck et al., 2001). Indeed, Bruinsma et al. (2003) have used the Mg II index for constructing their DTM2000 empirical model. On the other hand, Culot et al. (2004) have also shown that Mg II was a better proxy to model the red and green line emission at thermospheric altitudes during magnetic quiet periods.

Figure 7 (upper panel) shows the comparison between the CHAMP density $c_{1}$ projection coefficient (red line) and those computed with the actual (black line) and quiet (blue line) model using Mg II instead of F10.7 as a proxy for the Solar activity. For that purpose $\mathrm{Mg}$ II values have been scaled to F10.7 ones using the linear relationship found between these two quantities over 30 months (January 2002 to June 2005). In the NRLMSISE-00 model, the average value of the F10.7 index over three solar rotations is considered as an independent parameter that indeed is almost constant over May 2003. In our new model runs, we have used this mean F10.7 value as a constant parameter, that has been adjusted so that the 

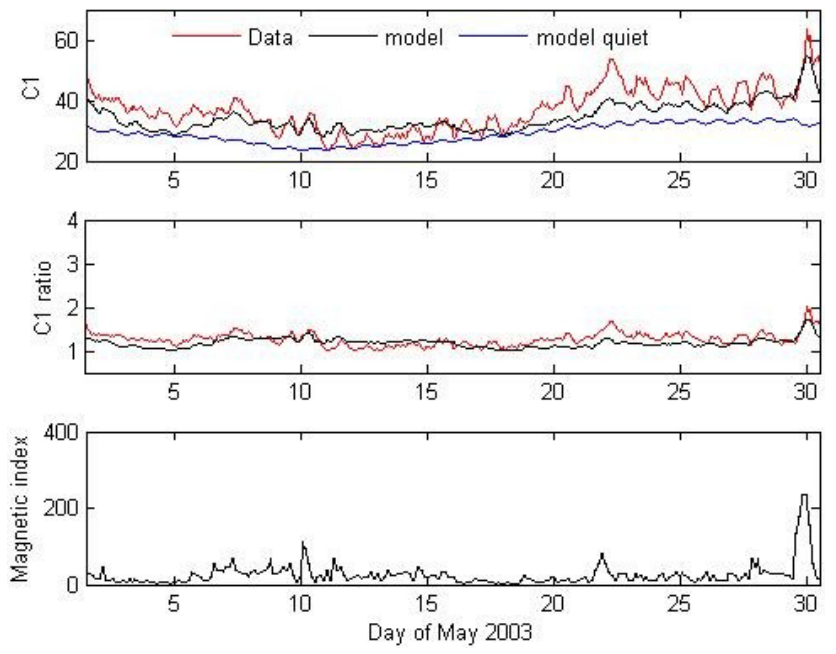

Fig. 7. Variations with UT of the $c_{1}$ projection coefficients for the NS orbit segments during May 2003.

Upper panel: variations of the CHAMP density $c_{1}$ projection coefficient (red line) and those computed with the actual (black line) and quiet (blue line) NRLMSISE-00 models using Mg II instead of F10.7 as a proxy for the solar activity.

Middle panel: variations of the $c_{1}$ (CHAMP) $/ c_{1}$ (NRLMSISE-00 quiet) (red line) and $c_{1}$ (NRLMSISE-00 actual) $/ c_{1}$ (NRLMSISE-00 quiet) (black line) ratios.

Lower panel: variations of the $a_{p}$ magnetic activity index.

CHAMP and model coefficients agree when there is no magnetic activity (i.e. when the black and blue curves overlap). This adjustment in fact accounts for any bias that could result from the CHAMP data reduction. It may also account for the fact that the model has not been constructed using the Mg II index.

One can see that, after this tuning of the model with regard to Solar activity, the $c_{1}$ projection coefficients for CHAMP and actual model densities are indeed in much better agreement than before (compare the upper panels of Figs. 5 and 7). For NS night time, and over the large range of magnetic activity that exist during the month of May 2003, the NRLMSISE-00 Mg II-tuned model (hereafter called actual $\mathrm{Mg}$ II or quiet Mg II models, depending on the used geomagnetic activity parameterization) reproduces quite well the large dynamic of the data variation.

However, this tuning does not significantly reduce the already mentioned important underestimation by the model of $c_{1}$ projection coefficients during periods of geomagnetic activity, including the intense storm that occurred at the end of the month. There is also a few day period (11 May to 15 May) that corresponds to a non-negligible overestimation of $c_{1}$ by the actual Mg II model. This period of small activity follows a long period of larger magnetic activity: this leads us to think that the model may overestimate the influence of the history of the magnetic activity.
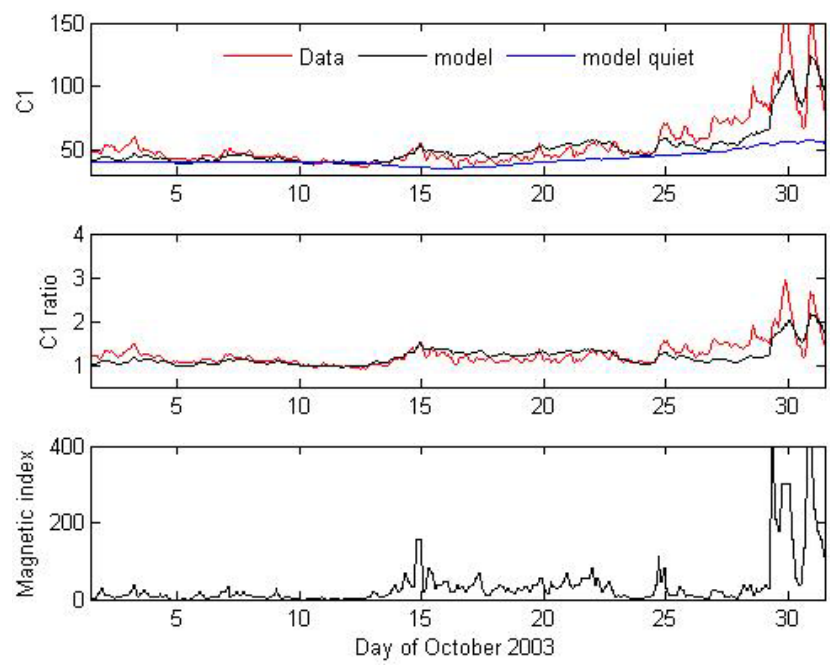

Fig. 8. Variations with UT of the $c_{1}$ projection coefficients for the SN orbit segments during October 2003.

Upper panel: variations of the CHAMP density $c_{1}$ projection coefficient (red line) and those computed with the actual (black line) and quiet (blue line) NRLMSISE-00 models using Mg II instead of F10.7 as a proxy for the solar activity.

Middle panel: variations of the $c_{1}(\mathrm{CHAMP}) / c_{1}$ (NRLMSISE-00 quiet) (red line) and $c_{1}$ (NRLMSISE-00 actual)/c $c_{1}$ (NRLMSISE-00 quiet) (black line) ratios.

Lower panel: variations of the $a_{p}$ magnetic activity index.

\subsection{A possible proxy}

This technique of modelling thermospheric densities has been applied to a more active period in October 2003, as shown in Fig. 8 (upper panel). The lower panel shows that this month was characterized by very low magnetic activity during its first half, and a severe storm, the so-called Halloween storm at the end of the month during which the $a_{p}$ value reaches 400 . Local time varies from the beginning to the end of the month from 3.7 to 1.1 for the night time SN segment shown here. CHAMP densities for the Halloween storm have been presented by Sutton et al. (2005), and we refer to this paper for the detailed study of this period. Note here that on 27 and 28 October, just prior to the intense storm, the CHAMP $c_{1}$ coefficient is already significantly larger than the quiet $\mathrm{Mg}$ II one.

May and October 2003 examples illustrate that, once tuned with regard to mean solar activity and use of the $\mathrm{Mg}$ II index, the NRLMSISE-00 model provides $c_{1}$ projection coefficient curves with variations that reproduce quite well those present in the CHAMP density $c_{1}$ curve, despite a non negligible underestimation of the magnitude of these variations.

Figures 7 and 8 (upper panels) also suggest that the $c_{1}$ projection coefficient computed with the quiet $\mathrm{Mg}$ II model would provide reference values for characterizing the variations in the thermospheric density that are driven by the geomagnetic activity. Indeed, the ratios between actual and 
quiet Mg II model $c_{1}$ coefficients (plotted with a black line on the middle panels of Figs. 7 and 8) would then represent the relative variations in the density that are associated to magnetic activity, as calculated by the model provided these ratios are independent of the other parameters. This can easily be checked by means of simulations for a satellite at a different altitude with the same values of the other parameters. For May 2003, we have verified that the ratio computed for the actual CHAMP situation and the corresponding one computed after setting the altitude at a constant value of $385 \mathrm{~km}$ are strictly identical (not shown here). Given the variability of the other parameters, this clearly shows that the quiet $\mathrm{Mg}$ II model $c_{1}$ projection coefficients provide reference values for characterizing the response of the thermospheric density to the geomagnetic forcing. We have thus used these values to calculate the CHAMP data relative variations associated to magnetic activity (red curve on middle panel of Figs. 7 and 8). Comparison with the black lines shows once more that the model characterization of magnetic activity perturbation can be improved. In a following paper, we will study these relative variations on a much longer time period and the possibility of nowcasting them will be discussed.

\section{Conclusion}

The SVD procedure presented in this paper allowed us to propose a new proxy for the global thermospheric response to auroral heating. The large scale spatial variations in the density, mostly related to altitude/latitude variations are captured by the first singular vector; time variations are captured by the associated projection coefficient, down to time scale on the order of one orbital period. Smaller scale spatial and time variations typically correspond to the variations induced by gravity waves generated by Joule heating as the one discussed by Forbes et al. (2006) and Bruinsma et al. (2006). Their morphology along each orbit segment is captured by the residual, i.e. the difference between the observed variation in the CHAMP density and its projection on the first singular vector. They can be large, as during the storm at the end of the month of May 2003, but they represent a small fraction of the total density variation, and we have not discussed them here.

We focus our study on time dependence of global scale variations in the thermospheric density. We show that the time variations in the projection coefficient do in fact represent those in the global density that are associated to magnetic activity as well as to solar EUV radiations. Therefore, we think that this projection coefficient may be of use in evaluating the accuracy of empirical thermosphere models.

We also show that the NRLMSISE-00 empirical model better accounts for the density forcing by solar radiations when tuned using the Mg II index as a proxy for solar EUV rather than the F10.7. Using the so modified model with a geomagnetic parameterization corresponding to a quiet geomagnetic situation provides reference values as far as geomagnetic activity is concerned. Such a reference quantity is needed to quantify the thermospheric density response to auroral energy deposition. Indeed the ratio c of CHAMP density projection coefficient to the quiet model projection coefficient is a global quantity, independent of altitude and latitude between $50 \mathrm{~N}$ and $50 \mathrm{~S}$ and in the range of the CHAMP altitudes, namely around $400 \mathrm{~km}$ (see Fig. 1). The accuracy of thermosphere models depends on the altitude, and so it is for the ratio c. It is likely to be still valid between say $300 \mathrm{~km}$ and $800 \mathrm{~km}$ where atomic oxygen is the main constituent. On the other hand, it has no meaning in auroral and polar zones. The $\mathrm{c}$ quantity therefore provides a proxy of the response of thermospheric density to geomagnetic activity forcing, and hence to space weather events for latitudes between $50 \mathrm{~N}$ and $50 \mathrm{~S}$ and with the best pertinence at altitudes around $400 \mathrm{~km}$.

\section{Appendix A}

In this appendix, we briefly recall the mathematical bases of the Singular Value Decomposition. We refer the reader to classical linear algebra treaties for demonstrations, and further mathematical developments (see, e.g., Horn and Johnson, 1985).

The so-called spectral theorem says that normal matrices can be unitarily diagonalized using a basis of eigenvectors. The SVD can be seen as a generalization of this theorem to any $m \times n$ matrix. It is based upon the following theorem:

Let $\mathbf{M}$ be an $m \times n$ matrix whose entries comes from the field $K$ (either real or complex numbers). Then there exists a factorization of the form

$\mathbf{M}=\mathbf{U} \Sigma \mathbf{V}^{*}$

Where:

- $\mathbf{U}$ is an $m \times m$ unitary matrix over $K$,

- $\Sigma$ is a $m \times n$ matrix, with nonnegative numbers $\Sigma_{k, k}=\lambda_{k}$ on the diagonal and zeros off the diagonal, and

- $\mathbf{V}^{*}$ denotes the conjugate transpose of $\mathbf{V}$, an $n \times n$ unitary matrix over $K$.

Such a factorization is called a singular-value decomposition of $\mathbf{M}$ :

- the matrix $\mathbf{V}$ contains a set of orthonormal "input" or "analysing" basis vector directions for $\mathbf{M}$

- the matrix $\mathbf{U}$ contains a set of orthonormal "output" basis vector directions for $\mathbf{M}$

- the matrix $\Sigma$ contains the singular values. 
If in addition the singular values $\lambda_{k}$ are ordered in nonincreasing fashion, the matrix $\Sigma$ is uniquely determined by $\mathbf{M}$, while it is not necessarily so for the matrices $\mathbf{U}$ and $\mathbf{V}$.

In the particular case where $\mathbf{M}$ is a Hermitian matrix having real and non-negative eigenvalues (i.e. $\mathbf{M}$ is positive semi-definite), the singular values and singular vector coincide with the eigenvalues and eigenvectors of $\mathbf{M}$. In the general case, Eq. (A1) leads to:

$$
\begin{aligned}
& \mathbf{M}^{*} \mathbf{M}=\mathbf{V} \Sigma^{*} \mathbf{U}^{*} \mathbf{U} \Sigma \mathbf{V}^{*}=\mathbf{V}\left(\Sigma^{*} \Sigma\right) \mathbf{V}^{*} \\
& \mathbf{M M}^{*}=\mathbf{U} \Sigma \mathbf{V}^{*} \mathbf{V} \Sigma^{*} \mathbf{U}^{*}=\mathbf{U}\left(\Sigma \Sigma^{*}\right) \mathbf{U}^{*}
\end{aligned}
$$

Consequently, the square of the non-zero singular values of $\mathbf{M}$ are equal to the non-zero eigenvalues of either $\mathbf{M}^{*} \mathbf{M}$ or $\mathbf{M M}^{*}$; the column vectors of $\mathbf{U}\left(\mathbf{u}_{k}\right.$, left singular vectors) are eigenvectors of $\mathbf{M} \mathbf{M}^{*}$ and the column vectors of $\mathbf{V}$ (right singular vectors) are eigenvectors of $\mathbf{M}^{*} \mathbf{M}$. Since $\mathbf{U}$ is an $m \times m$ unitary matrix, the left singular vectors $\mathbf{u}_{k}$ make an orthonormal basis on which the decomposition of any column vector of $\mathbf{M}$ is unique.

From a physical point of view, the $\mathbf{u}_{k}$ vectors can be interpreted in terms of the directions of the $K^{m}$ field that maximize the standard deviation of the data set along their direction, or equivalently minimize its inertia with respect to their direction. The $\lambda_{k}$ singular value is a measure of the standard deviation accounted for by the $\mathbf{u}_{k}$ singular vector. In our study we have normalized the singular values to $\Sigma\left(\lambda_{i}\right)^{2}$ (in other words, the normalized $\lambda_{i}$ values are such that $\left.\Sigma\left(\lambda_{i}\right)^{2}=1\right)$. Since the $\lambda_{k}$ values are ordered in nonincreasing fashion, $\mathbf{u}_{1}$ captures the maximum possible standard deviation of the sample on $K^{m}, \mathbf{u}_{2}$ captures the maximum possible standard deviation on the sample over the $K^{(m-1)}$ subspace perpendicular to $\mathbf{u}_{1}$, and so on.

In the present case, the SVD analysis is made on the set of 15 orbit segments corresponding to the considered orbit, the 7 previous ones and the 7 following ones. This is somehow a 15-orbit running SVD analysis over 15 consecutive orbit sections, i.e. a 24 -h long time window. The column vectors of matrix $\mathbf{M}$ are the $\boldsymbol{d}_{i}$ vectors whose elements $d_{i}\left(\varphi_{j}\right)$ are the density observed along orbit \#i at latitude $\varphi_{j}$ : the index $\mathrm{i}=1, \ldots, 15$ refers to the fifteen orbit segments during any 24-h UT period; the dimension of $\boldsymbol{d}_{i}$ is 101, corresponding to the 101 latitude degrees between $50 \mathrm{~N}$ and $50 \mathrm{~S}$. $\mathrm{M}$ is then a $101 \times 15$ matrix (i.e. $m=101 ; n=15$ ). Since the $\boldsymbol{u}_{k}$ vectors make an orthonormal basis on $K^{m}$, any column vector $\boldsymbol{d}_{i}$ of M can be expressed as:

$\boldsymbol{d}_{i}=\sum_{k=1}^{m} c_{i k} \boldsymbol{u}_{k}$

where $c_{i k} \boldsymbol{u}_{k}$ is the projection of the observed density profile $\boldsymbol{d}_{i}$ on the $k$-th singular vector $\boldsymbol{u}_{k}: c_{i k}$ is equal to the scalar product $\boldsymbol{d}_{i} \cdot \boldsymbol{u}_{k}$ between $\boldsymbol{d}_{i}$ and $\boldsymbol{u}_{k}$. Since $\boldsymbol{u}_{k}$ is a normalized vector $\left(\left\|\boldsymbol{u}_{k}\right\|=1\right)$, it is dimensionless, and the $\mathrm{c}_{1}$ coefficients have the dimension of a density.
Acknowledgements. This research has been supported by the French CNRS/INSU Programme National Soleil-Terre. The authors thank an anonymous referee for his constructive comments that helped us to improve the paper.

Topical Editor U.-P. Hoppe thanks T. Dudok de Wit and another anonymous referee for their help in evaluating this paper.

\section{References}

Bruinsma, S., Forbes, J. M., Nerem, R. S., and Zhang, X.: Thermosphere density response to the 20-21 November, 2003 Solar and geomagnetic storm from CHAMP and GRACE accelerometer data, J. Geophys. Res., 111, A06303, doi:10.1029/2005JA011284, 2006.

Bruinsma, S., Tamagan, D., and Biancale, R.: Atmopheric densities derived from CHAMP/STAR accelerometer observation, Planet. Space Sci., 52, 297-312, 2004.

Bruinsma, S., Thuillier, G., and Barlier, F.: The DTM-2000 empirical thermosphere model with new data assimilation and constraints at lower boundary, accuracy and properties, J. Atmos. Sol. Terr. Phys., 65, 1053-1070, 2003.

Burns, A. G., Killeen, T. L., Deng, W., Carignan, G. R., and Roble, R. G.: Geomagnetic storm effects in the low to middle upper thermosphere, J. Geophys. Res., 100, 14673-14 691, 1995.

Culot, F., Lathuillère, C., Lilensten, J., and Witasse, O.: The OI $630 \mathrm{~nm}$ and $557.7 \mathrm{~nm}$ dayglow measured by WINDII and modeled by TRANSCAR, Ann. Geophys., 22, 1947-1960, 2004, http://www.ann-geophys.net/22/1947/2004/.

Forbes, J. M., Lu, G., Bruinsma, S., Nerem, R. S., and Zhang, X.: Thermosphere density variation due to the April 15-24, 2002 Solar event from CHAMP/STAR accelerometer measurements, J. Geophys. Res., 110, A12S27, doi:10.1029/2004JA010856, 2006.

Hanuise, C., Cerisier, J. C., Auchère, F., Bocchialini, K., Bruinsma, S., Cornilleau-Wehrlin, N., Jakowski, N., Lathuillère, C., Menvielle, M., Valette, J.-J., Vilmer, N., Watermann, J., and Yaya, P.: From the Sun to the Earth: impact of the 27-28 May 2003 solar events on the magnetosphere, ionosphere and thermosphere, Ann. Geophys., 24, 129-151, 2006, http://www.ann-geophys.net/24/129/2006/.

Horn, R. A. and Johnson, C. R.: "Matrix Analysis", Section 7.3, Cambridge University Press, 1985.

Killeen, T. L., Won, Y. I., Niciejewski, R. J., and Burns, A. G.: Upper thermospheric winds and temperatures in the geomagnetic polar cap: Solar cycle, geomagnetic activity, and interplanetary magnetic field dependencies, J. Geophys. Res., 100, $21237-21342,1995$.

Lathuillère, C. and Menvielle, M.: WINDII thermosphere temperature perturbation for magnetically active situations, J. Geophys. Res., 109, A11304, doi:10.1029/2004JA010526, 2004.

Liu, H., Lühr, H., Henize, V., and Köhler, W.: Global distribution of the thermospheric total mass density derived from CHAMP, J. Geophys. Res., 110, A04301, doi:10.1029/2004JA010741, 2005.

Picone, J. M., Hedin, A. E., Drob, D. P., and Aikin, A. C.: NRLMSISE-00 empirical model of the atmosphere: Statistical comparisons and scientific issues, J. Geophys. Res., 107, 1468, doi:10.1028/2002JA009430, 2002.

Reigber, C., Lühr, H., and Schwintzer, P.: CHAMP mission status, Adv. Space Res., 30, 129-134, 2002. 
Rhoden, E., Forbes, J. M., and Marcos, F. A.: The influence of geomagnetic and solar variabilities on lower thermospheric density, J. Atmos. Sol. Terr. Phys., 62, 999-1013, 2000.

Sutton, E. K., Forbes, J. M., and Nerem, R. S.: Global thermospheric neutral density and wind response to the severe 2003 geomagnetic storms from CHAMP accelerometer data, J. Geophys. Res., 110, A09S40, doi:10.1029/2004JA010985, 2005.

Thuillier, G. and Bruinsma, S.: the Mg II index for upper atmosphere modelling, Ann. Geophys., 19, 219-228, 2001,

http://www.ann-geophys.net/19/219/2001/.
Viereck, R. A., Puga, L., McMullin, D., Judge, D., Weber, M., and Tobiska, W. K.: The Mg II Index: A proxy for solar EUV, Geophys. Res. Lett., 28, 7, 1343-1346, 2001.

Viereck, R. A., Floyd, L. E., Crane, P. C., Woods, T. N., Knapp, B. G., Rottman, G., Weber, M., Puga, L. C., and Deland, M. T.: A composite Mg II index spanning from 1978 to 2003, Space Weather, 2, S10005, doi:10.1029/2004SW0000084, 2004. 